\title{
Familiarity is related to conceptual implicit memory: An examination of individual differences
}

\author{
Wei-chun Wang • Andrew P. Yonelinas
}

Published online: 26 July 2012

(C) Psychonomic Society, Inc. 2012

\begin{abstract}
Explicit memory is thought to be distinct from implicit memory. However, growing evidence has indicated that explicit familiarity-based recognition memory judgments rely on the same process that supports conceptual implicit memory. We tested this hypothesis by examining individual differences using a paradigm wherein we measured both familiarity and conceptual implicit memory within the same participants. In Experiments 1a and $1 \mathrm{~b}$, we examined recognition memory confidence ROCs and remember/know responses, respectively, to estimate recollection and familiarity, and used a free association task to measure conceptual implicit memory. The results demonstrated that, across participants, familiarity, but not recollection, was significantly correlated with conceptual priming. In contrast, in Experiment 2, utilizing a similar paradigm, a comparison of recognition memory ROCs and explicit associative cued-recall performance indicated that cued recall was related to both recollection and familiarity. These results are consistent with models assuming that familiarity-based recognition and conceptual implicit memory rely on similar underlying processes.
\end{abstract}

Keywords Familiarity · Recollection · Implicit memory · Conceptual priming $\cdot$ Associative cued recall

It has long been thought that declarative, or explicit, memory is supported by processes reliant on the medial temporal lobes,

W.-c. Wang $(\bowtie) \cdot$ A. P. Yonelinas

Department of Psychology, University of California, Davis, One Shields Avenue,

Davis, CA 95616, USA

e-mail:wwa@ucdavis.edu

A. P. Yonelinas

Center for Mind and Brain, University of California, Davis,

Davis, CA, USA whereas nondeclarative, or implicit, memory is supported by neocortical regions (e.g., Gabrieli, 1998; Schacter, Chiu \& Ochsner, 1993; Squire, 2004). An alternative possibility, however, is that familiarity-based recognition and implicit memory rely on a common underlying process (e.g., Henke, 2010; Jacoby, 1991; Mandler, 1980; Wagner \& Gabrieli, 1998; Yonelinas, 2002). It has, for example, been suggested that the same process that leads an item to seem familiar on an explicit recognition memory test may also lead an item to come to mind more readily in a conceptual implicit memory test, such as an implicit exemplar generation task (Henke, 2010; Wagner \& Gabrieli, 1998; Wang, Lazzara, Ranganath, Knight \& Yonelinas, 2010; Yonelinas, 2002; but see Donaldson, Petersen, \& Buckner, 2001; Voss, Lucas, \& Paller, 2012).

Consistent with the hypothesis that conceptual implicit memory and familiarity are supported by the same underlying process, both are sensitive to behavioral manipulations of attention, study duration, and depth of encoding (e.g., Challis \& Sidhu, 1993; Hamann, 1990; Light, Prull \& Kennison, 2000; Mulligan \& Stone, 1999; Srinivas \& Roediger, 1990; for a review, see Yonelinas, 2002). In contrast, perceptual implicit memory, as measured on tasks such as word fragment completion, is generally unaffected by these manipulations, suggesting that these forms of implicit memory are distinct (Gabrieli, 1998; Schacter et al., 1993). Moreover, patient and neuroimaging studies have demonstrated that the perirhinal cortex, a medial temporal lobe region adjacent to the hippocampus, is critical for both familiarity (Yonelinas, Kroll, Quamme, Lazzara, Sauvé, Widaman \& Knight, 2002; for reviews, see Diana, Yonelinas \& Ranganath, 2007; Eichenbaum, Yonelinas \& Ranganath, 2007; Yonelinas, Aly, Wang \& Koen, 2010) and conceptual implicit memory (e.g., Blaxton, 1992; O'Kane, Insler \& Wagner, 2005; Voss, Hauner, \& Paller, 2009; Wang et al., 2010; but see Levy, Stark \& Squire, 2004).

In the present study, we adopted a novel approach to test the hypothesis that conceptual implicit memory and familiarity rely on the same underlying process. Rather than 
examining how these types of memory respond to different experimental variables, or looking to determine the brain regions involved in these processes, we sought to investigate whether participants with greater familiarity would exhibit more conceptual implicit memory than did participants with less familiarity. In the experiments reported below, participants incidentally encoded a word list by judging whether each word was abstract or concrete. Afterward, they were given a recognition memory test containing studied words intermixed with new words and asked to make either recognition confidence or remember/know judgments that were used to derive recollection and familiarity estimates (see Yonelinas, 1994, 1999, 2001). In the last phase, participants completed an implicit free association task in which they were presented with nonstudied cue words and asked to produce the first related word that came to mind. These words were selected to be associated with specific target words, some of which had been studied and some of which had not. Priming was measured as the proportion of studied targets generated relative to unstudied target, or baseline, words.

If, consistent with the behavioral and neural evidence, the same process supports both conceptual priming and familiarity, we would then expect these forms of memory to be positively correlated across participants. In contrast, we do not expect to see a strong relationship between implicit memory and recognition responses that were based on recollection, because recollection is generally associated with processes supported by the hippocampus rather than the perirhinal cortex (for reviews, see Diana et al., 2007; Eichenbaum et al., 2007; Yonelinas et al., 2010). In Experiment 1a, we assessed recollection and familiarity using a receiver operating characteristic (ROC) confidence rating method, whereas in Experiment $1 \mathrm{~b}$ we tested the generalizability of these results using a remember/know method. For Experiment 2, we replaced the implicit test with a comparable explicit cuedrecall test to determine how recollection and familiarity were related to explicit recall.

\section{Experiment 1}

\section{Experiment 1a}

\section{Method}

Participants and materials A total of 53 undergraduates participants (mean age $=19.83$ years, $S D=2.35$; mean education $=13.53$ years, $S D=1.49 ; 37$ female, 16 male) were recruited from the psychology participant pool at the University of California, Davis, in exchange for course credit. Five of the participants were excluded from the data analysis: one due to software malfunction and four because of chance performance in the recognition test.
The materials consisted of 320 cue-target word pairs with a mean forward association strength of $.38(S D=.11)$ (i.e., for a given cue, the target has a $38 \%$ chance of being produced). The word pairs were compiled from the Nelson, McEvoy, and Schreiber (1998) database and divided into four lists of 80 pairs each for counterbalancing purposes. Each list served in each of the four conditions described below. All stimuli were presented, and keyboard responses recorded, on a Dell PC. Verbal responses from the free association task were digitally recorded and transcribed.

Design and procedure First, participants incidentally encoded 160 target words by judging whether the words were abstract (e.g., EFFECT) or concrete (e.g., LEMON). Each word was presented for $1,200 \mathrm{~ms}$, with a $200-\mathrm{ms}$ fixation cross between stimuli. In the second phase, participants completed a surprise recognition memory test for 80 studied (e.g., LEMON) and 80 unstudied (e.g., CLAY) targets. For each word, participants responded on a 6-point confidence scale, from 1 (sure new) to 6 (sure old). They were allotted 1,500 ms for each recognition judgment, with a 500-ms fixation cross between words.

In the final phase, participants were given a free association task wherein they were presented with all 320 unstudied word cues. For each cue, participants were instructed to respond verbally with the first strongly associated word that came to mind. Each cue was presented on screen for $3,000 \mathrm{~ms}$, with a $1,500-\mathrm{ms}$ fixation cross between stimuli. One set of 80 cue words was paired with targets that had been in both the encoding and recognition phases (EncRec; e.g., LIME-LEMON). Another set was paired with targets that had appeared only during the encoding phase (Enc; e.g., CAUSE-EFFECT). A third set was paired with targets that appeared only in the recognition phase (Rec; e.g., POTTERY-CLAY). The remaining 80 cues were paired with the unstudied targets, which had not appeared in the experiment (e.g., FORTUNE-FAME). These unstudied pairs served as a baseline, and the differences between the baseline generation rate and the generation rates for the other three conditions served as measures of conceptual implicit memory. Following the free association task, participants were administered an awareness questionnaire to assess whether they utilized explicit strategies during the task (adapted from Bowers \& Schacter, 1990).

\section{Results}

Recognition confidence ratings were used to plot ROCs that were fitted by minimizing the sum of squared errors in the dual-process signal detection (DPSD) model to derive recollection and familiarity estimates (see Yonelinas, 1994, 1999, 2001; the average ROC is presented in Fig. 1a). The 
Fig. 1 Recognition memory performance in all three experiments. a Average receiver operating characteristic curves from Experiment 1a and 2, fit to the dual-process signal detection model. Chance performance is represented by the diagonal. b Overall recognition memory discriminability $\left(d^{\prime}\right)$, recollection, and familiarity in all three experiments a

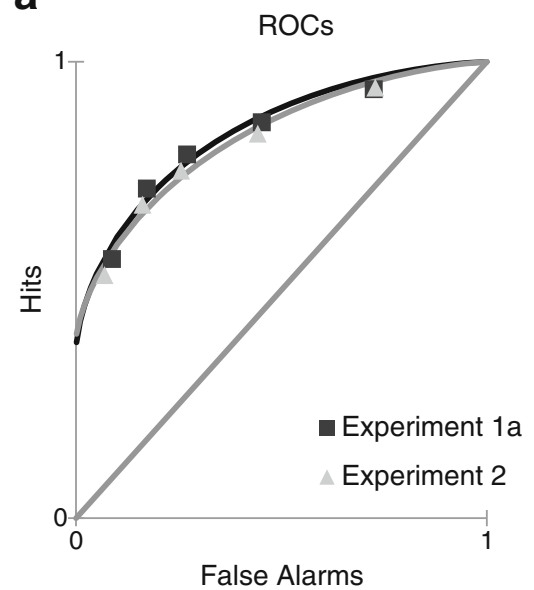

b

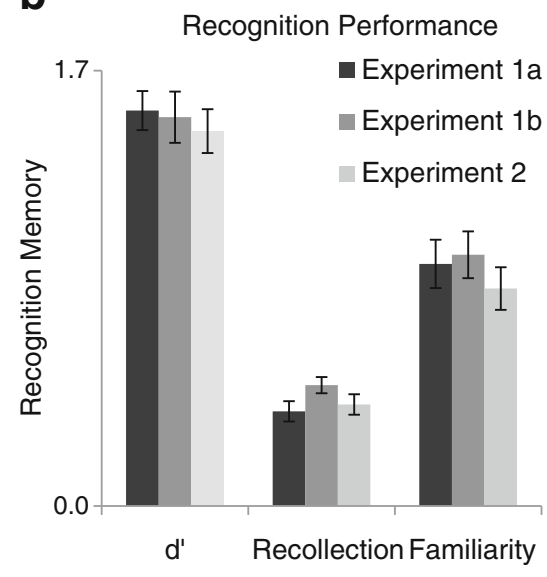

leftmost point in the figure reflects the proportion of old items receiving a " 6 " response (i.e., hits) against the proportion of new items receiving a "6" response (i.e., false alarms). Each consecutive point reflects a more lax scoring criterion (i.e., in the second point, both " 6 "s and "5"s are treated as hits and false alarms). The ROC for each participant was fit with the DPSD model to estimate the contributions of recollection, which approximates the $y$-intercept, and familiarity, which is related to the curvilinearity of the function. The individual ROCs were consistent with those of prior studies, and there was little evidence of floor, ceiling, truncation, or limited-range effects that could have compromised the analysis (Yonelinas \& Parks, 2007). The average probability estimate of recollection was $.37(S D=.28)$, and the average familiarity $d$ ' measure was $0.95(S D=0.65)$.

Conceptual implicit memory was measured as the proportion of baseline targets generated relative to the proportion of studied targets generated (Table 1). Studied items were broken down into those that had been presented in both the encoding and recognition phases (EncRec), those presented only in the encoding phase (Enc), and those presented only in the recognition test (as lure items, Rec). In order to investigate the relationship between recognition and conceptual implicit memory, the recollection and familiarity estimates were correlated with each priming measure. Because there were no significant differences in the correlations between the priming and explicit memory measures, we will only describe the correlations with average priming.

We first examined whether overall recognition discriminability (as measured using $d$ ' at the midpoint confidence level; $M=1.55, S D=0.53$; Fig. $1 \mathrm{~b}$ ) was positively related to conceptual priming, and found that participants with higher recognition also had higher priming $[r(46)=.44, p<.005$; Fig. 2a]. More importantly, consistent with our prediction, recollection did not significantly correlate with priming $[r$ (46) $=-.15, p=.31]$, whereas familiarity was strongly correlated with priming $[r(46)=.47, p<.001]$. Additionally, these two correlations were significantly different, indicating that familiarity has a stronger relationship with priming than does recollection $[t(45)=2.58, p<.05]$.

To determine whether the correlation between familiarity and priming was due to the use of explicit memory retrieval during the implicit test, we conducted an additional analysis wherein we excluded participants who reported using
Table 1 Experiment 1a: Generation rates for three conceptual priming conditions, the average, and baseline; proportion priming for the three conditions and average priming; zero order correlations between all three priming conditions and average priming and $d^{\prime}$, recollection, and familiarity; partial correlations between all three priming conditions and average priming and recollection and familiarity

\begin{tabular}{|c|c|c|c|c|c|c|c|}
\hline & \multirow[t]{2}{*}{$M(S D)$} & \multirow[t]{2}{*}{ Priming $(S D)$} & \multicolumn{3}{|c|}{ Correlation With Priming } & \multicolumn{2}{|c|}{ Partial Correlation } \\
\hline & & & $d^{\prime}$ & $\mathrm{R}$ & $\mathrm{F}$ & $\mathrm{R}$ & $\mathrm{F}$ \\
\hline EncRec & $.42(.11)$ & $.10(.09)$ & $.31^{*}$ & -.16 & $.42^{*}$ & .11 & $.40^{*}$ \\
\hline Enc & $.39(.10)$ & $.06(.09)$ & $.47^{*}$ & -.14 & $.48^{*}$ & .20 & $.50^{*}$ \\
\hline $\operatorname{Rec}$ & $.38(.09)$ & $.06(.08)$ & $.49^{*}$ & -.10 & $.36^{*}$ & .14 & $.37^{*}$ \\
\hline Average & $.40(.09)$ & $.07(.08)$ & $.44^{*}$ & -.15 & $.48^{*}$ & .18 & $.48^{*}$ \\
\hline Baseline & $.33(.09)$ & & & & & & \\
\hline
\end{tabular}

EncRec, words presented in both the encoding and recognition phases; Enc, words presented only at encoding; Rec, words presented only in the recognition test. ${ }^{*} p<.05$. 
Fig. 2 Average conceptual priming (Exps. 1a and 1b) and cued recall (Exp. 2) plotted against measures of recognition memory. Recollection and familiarity were estimated with ROCs in Exps. 1a and 2, whereas the remember/know method was used in Exp. 1b. a In Experiment 1a, $d^{\prime}$ and familiarity, but not recollection, increased with conceptual priming. b Similarly, $d^{\prime}$ and familiarity, but not recollection, were correlated with conceptual priming in Experiment 1b. $\mathbf{c}$ In Experiment 2, $d$ ' and recollection, but not familiarity, were related to cued recall. ${ }^{*} p<.05$
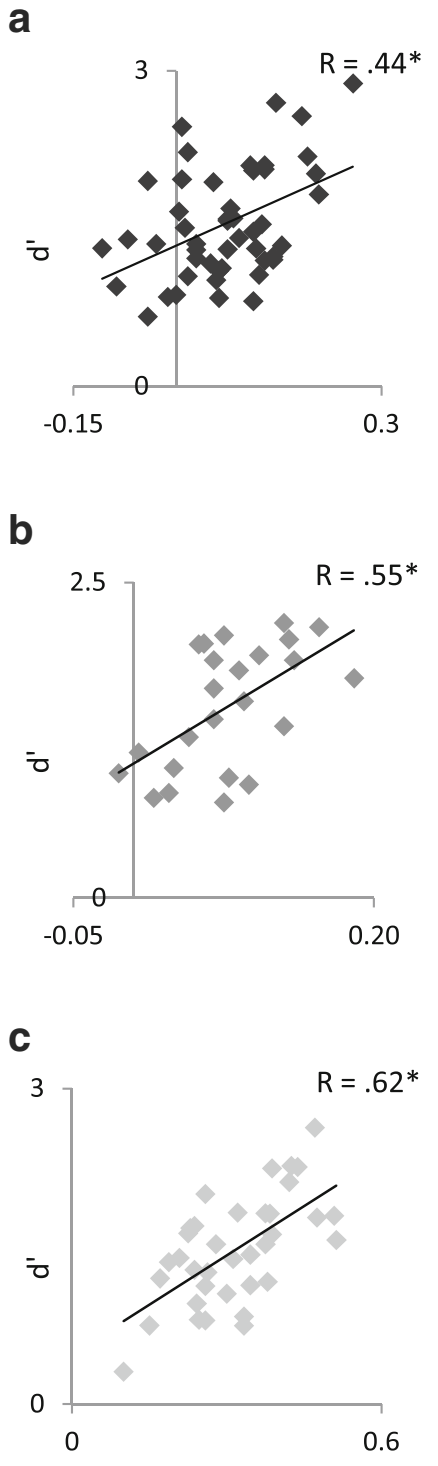

explicit retrieval strategies during the free association task (i.e., participants who reported "actively [trying] to remember words" from the study phases). The results indicated that when explicitly contaminated participants were removed for the analysis, familiarity was still significantly correlated with conceptual priming $[r(29)=.40, p<.05]$.

A subsequent analysis indicated that recollection and familiarity estimates were negatively correlated across participants $[r(46)=-.58, p<.001]$. In general, we have found that these two parameters are not correlated, but that they can become negatively correlated when the number of trials making up a ROC is low (e.g., because there were several necessary conditions in the present study, we included only 80 old trials in each participant-condition). We were therefore concerned that the lack of a correlation between implicit memory and recollection might have been related to a measurement artifact inherent in the correlation analysis that

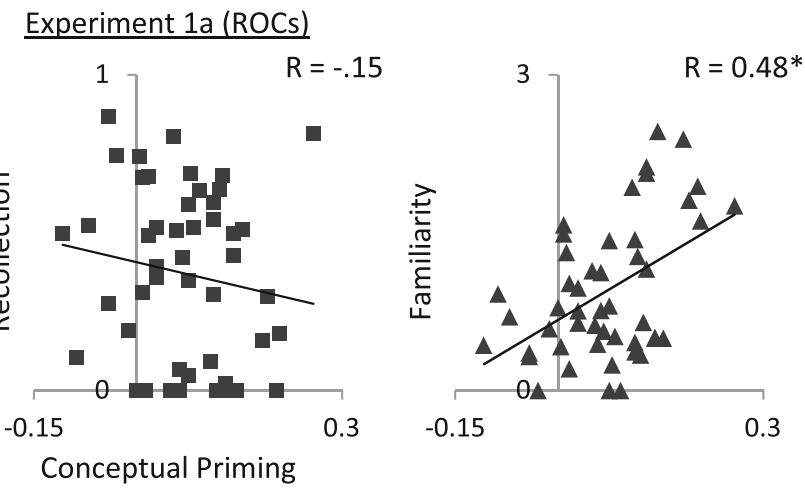

Experiment $1 \mathrm{~b}(\mathrm{R} / \mathrm{K})$
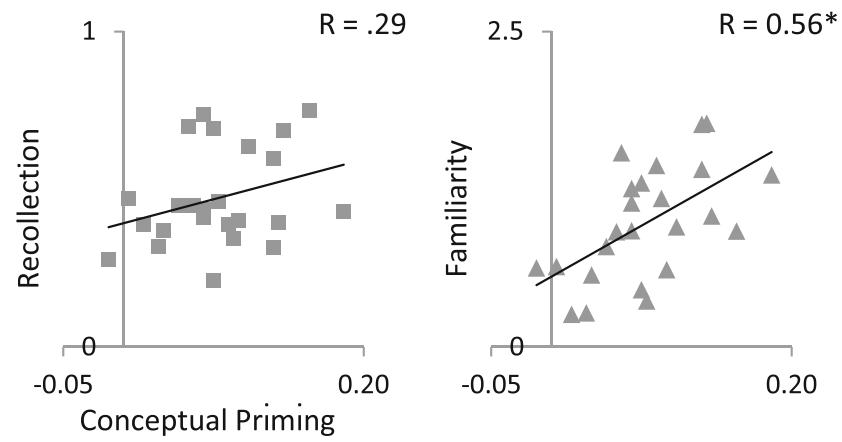

Experiment 2 (ROCs)
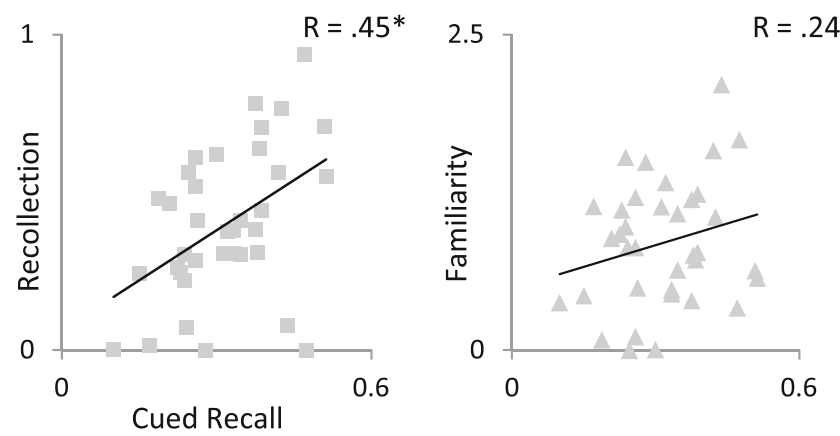

may have masked a true correlation. To address this concern, we first analyzed partial correlations to reveal potential mediation or suppression effects. Consistent with the zeroorder correlations, recollection was not significantly correlated with priming when controlling for familiarity $[r(45)=$ $.18, p=.23$ ], whereas familiarity was significantly correlated with priming when controlling for recollection $[r(45)=$ $.48, p<.001]$. Second, to assess the possibility that the null relationship between recollection and conceptual priming was due to the fact that recollection and familiarity were collinear, we assessed multicollinearity measures in a meancentered regression $\left[R^{2}=.25, R_{\mathrm{adj}}^{2}=.22 ; F(2,45)=7.57\right.$, $p<.001]$ with recollection and familiarity predicting conceptual implicit memory. Consistent with the correlations, familiarity significantly predicted priming $[\beta=.59 ; t(45)=$ $3.71, p<.001]$, while recollection did not $[\beta=.20 ; t(45)=$ $1.22, p=.23]$, and multicollinearity measures were within 
acceptable ranges $\left(\mathrm{VIF}=1.51,{ }^{1} \kappa=6.44^{2}\right)$. Thus, we found little evidence that the correlations that we observed were a product of measurement artifacts. For simulations further examining the potential effects of subsampling on parameter correlations, see Appendix A.

The conclusion that priming is related to familiarity and not recollection relies on the ROC analysis. Although a large body of evidence has supported the validity of that approach (e.g., Yonelinas et al., 2010), the method relies on numerous assumptions that can be questioned (see, e.g., Wixted, 2007). Thus, to provide a further test of the results, in Experiment $1 \mathrm{~b}$ we addressed the same question but utilized an alternative method of estimating recollection and familiarity: the remember/know procedure (Gardiner, 1988; Tulving, 1985). Although the assumptions of any one procedure may be questioned, if multiple measurement methods converge on the same results, one can be more confident in the conclusions.

\section{Experiment $1 \mathrm{~b}$}

\section{Method}

Participants and materials A group of 29 undergraduate participants (mean age $=19.63$ years, $S D=1.17$; mean education $=14.04$ years, $S D=1.12 ; 18$ female, 11 male) were recruited from the University of California, Davis, psychology participant pool. Five of the participants were excluded from the data analyses: three due to experimenter error, one for chance recognition performance, and one for not completing the free association task. The materials were identical to those of Experiment 1a.

Design and procedure The procedure was also identical to that of Experiment 1a, except that the recognition confidence judgments were replaced with remember/know judgments. Rather than instructing participants to respond on a 6-point confidence scale, they were required to respond with a "remember," "familiar," or "new" judgment for each presented word. Participants were instructed to respond "remember" when they could, if asked, tell the experimenter the specific details(s) that they recollected when they saw the word (e.g., what it looked like on the screen or their thoughts and feelings at the time). Participants were instructed to respond "familiar" if they believed that the word was previously studied, but were unable to recollect any specific details about the study event. Finally,

\footnotetext{
${ }^{1}$ The variance inflation factor (VIF) is a measure of how much variance is increased because of collinearity. The square root of the VIF indicates how much the standard error has been inflated for each independent variable.

${ }^{2}$ The condition number $(\kappa)$ is the largest condition index, which is a measure of the collinearity in the combinations of independent variables in the model.
}

participants were instructed to respond "new" if they thought that the word was not previously studied. Participants explained their responses to the experimenter during the practice phase to ensure that they understood the instructions. Note that recent studies have indicated that these instructions are necessary to ensure that participants do not confuse remember/know instructions with confidence instructions (Rotello, Macmillan, Reeder \& Wong, 2005; Yonelinas, 2001; Yonelinas \& Parks, 2007). The only other change relative to the previous experiment was that participants were given an extra second for each recognition judgment (i.e., $2,500 \mathrm{~ms}$ ), with a 500 -ms fixation cross between words.

\section{Results}

Recollection was estimated as the probability of a "remember" response to an old item minus the probability of a "remember" response to a new item. Familiarity was estimated as the difference in the probability of a "familiar" response given that the item was not remembered [i.e., "familiar"/(1 - "remember")] for old and new items. Familiarity discriminability was measured as $d$, given the familiarity estimates for old and new items (see Yonelinas \& Jacoby, 1995). Again, similar to the previous experiment, we compared mean recollection $(M=.47, S D=.15$; Fig. $1 \mathrm{~b})$ and familiarity $(M=.98, S D=.45)$ to average free association priming $(M=.08, S D=.05)$, given that the correlations across the implicit memory conditions did not differ (Table 2).

First, overall recognition discriminability (as measured by $d^{\prime}: M=1.52, S D=0.49$ ) was positively correlated with conceptual priming $[r(22)=.55, p<.01$; Fig. 2b]. Moreover, consistent with Experiment 1a, priming was positively correlated with familiarity $[r(22)=.59, p<.005]$, but not with recollection $[r(22)=.29, p=.17]$. These two correlations, however, were not significantly different $[t$ $(21)=1.21, p=.12]$.

Unlike Experiment 1a, where recollection and familiarity were negatively correlated, they were unrelated in the present experiment $[r(22)=.22, p=.30]$. Thus, there was no concern that the observed correlation between familiarity and priming was an artifact produced by the negative familiarity-recollection correlation. To be thorough, however, we conducted partial correlation and regression analyses to examine any potential mediation, suppression, or collinearity effects. Consistent with the zero-order correlations, conceptual priming was not correlated with recollection when controlling for familiarity $[r(21)=.20, p=.36]$, whereas it was significantly correlated with familiarity when controlling for recollection $[r(21)=.56, p<.005]$. Additionally, the mean-centered regression $\left[R^{2}=.37, R_{\text {adj }}{ }^{2}=.31 ; F(2,21)=\right.$ 
Table 2 Experiment 1b: Generation rates for three conceptual priming conditions, the average, and baseline; proportion priming for the three conditions and average priming; zero order correlations between all three priming conditions and average priming and $d$, recollection, and familiarity; partial correlations between all three priming conditions and average priming and recollection and familiarity

\begin{tabular}{|c|c|c|c|c|c|c|c|}
\hline & \multirow[t]{2}{*}{$M(S D)$} & \multirow[t]{2}{*}{ Priming $(S D)$} & \multicolumn{3}{|c|}{ Correlation With Priming } & \multicolumn{2}{|c|}{ Partial Correlation } \\
\hline & & & $d^{\prime}$ & $\mathrm{R}$ & $\mathrm{F}$ & $\mathrm{R}$ & $\mathrm{F}$ \\
\hline EncRec & $.44(.11)$ & $.12(.07)$ & $.49^{*}$ & .29 & $.42^{*}$ & .22 & $.38^{\dagger}$ \\
\hline Enc & $.39(.09)$ & $.06(.06)$ & $.36^{\dagger}$ & .24 & $.39^{\dagger}$ & .17 & $.35^{\dagger}$ \\
\hline $\operatorname{Rec}$ & $.39(.08)$ & $.06(.06)$ & $.46^{*}$ & .19 & $.56^{*}$ & .09 & $.54^{*}$ \\
\hline Average & $.41(.09)$ & $.08(.05)$ & $.55^{*}$ & .29 & $.59^{*}$ & .20 & $.56^{*}$ \\
\hline Baseline & $.33(.07)$ & & & & & & \\
\hline
\end{tabular}

EncRec, words presented in both the encoding and recognition phases; Enc, words presented only at encoding; Rec, words presented only in the recognition test. ${ }^{\dagger} p<.05$, one tailed; ${ }^{*} p<.05$, two tailed.

$6.20, p<.01]$ indicated that familiarity significantly predicted priming $[\beta=.55 ; t(21)=3.11, p<.005]$, while recollection did not $[\beta=.17 ; t(21)=0.94]$, and that multicollinearity measures were within acceptable ranges (VIF $=$ $1.05, \kappa=7.88)$.

\section{Experiment 1 Discussion}

The results of Experiments $1 \mathrm{a}$ and $1 \mathrm{~b}$ indicated that conceptual implicit memory was positively correlated with familiarity, but not with recollection. This pattern was observed in the correlation and regression analyses, and was observed when recollection and familiarity were measured using the confidence-based ROC method, as well as with the remember/know method.

One concern with Experiment 1a was that there was a negative correlation between recollection and familiarity estimates across participants. However, subsequent analyses suggested that this did not greatly impact the findings, and Experiment $1 \mathrm{~b}$ showed that the same pattern of results was observed under conditions in which there was no negative correlation between recollection and familiarity. Thus, the results indicate that the relationship between familiarity and conceptual implicit memory is quite general and is not likely due to artifacts related to collinearity or to the particular method used to estimate recollection and familiarity.

Nonetheless, we had one further concern about the initial results; the lack of a correlation between recollection and implicit memory might have arisen because the familiarity parameter in the ROC and remember/know experiments may be a generally better memory measure, and thus it may always be more strongly related to other memory measures than recollection. To test this possibility, we conducted Experiment 2, wherein the implicit free association task was replaced with an explicit associative cued-recall task. Under these conditions, we expected to see a correlation between the recollection parameter and the recall measure, on the basis that explicit cued recall should rely more on recollection than would an implicit task (Mandler, 1980). In contrast, if the null relationship between recollection and priming seen in Experiments 1a and $1 \mathrm{~b}$ reflected the fact that our recollection estimate was less related to other memory measures than was familiarity, then recollection should not be correlated with explicit associative cued-recall performance.

A secondary question that the experiment allowed us to address was how familiarity was related to explicit cued recall. One prior study had found that recollection, but not familiarity, correlated with free recall performance (Quamme, Yonelinas, Widaman, Kroll \& Sauvé, 2004). However, there is evidence that associative cued recall is related to "feelings of knowing" (Gruneberg \& Monks, 1974) and that it can be influenced by both controlled recollective and automatic familiarity processes (McCabe, Roediger \& Karpicke, 2011). Moreover, both recollection and familiarity are correlated with associative recognition, which is thought to depend more on recollection (Starns \& Ratcliff, 2008). To the extent that the processing of a word leads to activation of its associates, one might expect that this could increase associative cuedrecall performance. Thus, familiarity may also be related to associative cued recall, though to a lesser extent than recollection.

\section{Experiment 2}

Method

Participants and materials A group of 38 undergraduate participants (mean age $=19.16$ years, $S D=1.87$; mean education $=12.97$ years, $S D=1.48 ; 26$ female, 12 male) were recruited from the University of California, Davis, psychology participant pool. Two of the participants were excluded from the data analyses: one for not being a native English speaker, and the other for chance recognition performance. The materials were identical to those of Experiments 1a and $1 \mathrm{~b}$. 
Design and procedure The procedure was also identical to that of Experiment 1a, except that the free association instructions were replaced with explicit associative cuedrecall instructions. Rather than responding with the first related word that came to mind for a given cue, participants were told to think back to the previous encoding and recognition phases and to respond only with a previously studied target that was associated with the given cue.

\section{Results}

The ROCs were examined as in Experiment 1a, and the average ROC is presented in Fig. 1a.

Mean recollection $(M=.40, S D=.24)$ and familiarity $(M=$ $.85, S D=.50$ ) estimates were derived from the DPSD model for each individual participant. Cued recall was measured as the proportion of unstudied targets recalled (i.e., false recall) subtracted from the proportion of studied targets generated from each condition, yielding three measures of cued recall, as well as an average (Table 3). As in Experiment 1, the correlations between the recognition parameters and the three recall measures were similar, so we focus here on the correlations with average recall.

Overall recognition discriminability (as measured by $d$ : $M=1.47, S D=0.51$; Fig. $1 \mathrm{~b}$ ) was significantly correlated with cued recall $[r(34)=.62, p<.001$; Fig. $2 c]$. In contrast to Experiments $1 \mathrm{a}$ and $1 \mathrm{~b}$, recall was significantly correlated with recollection $[r(34)=.45, p<.01]$, but not with familiarity $[r(34)=.23, p=.18]$. These two correlations, however, were not significantly different $[t(33)=0.85]$.

As in Experiment 1a, recollection and familiarity were negatively correlated $[r(34)=-.40, p<.05]$. An examination of the partial correlations indicated that cued recall was correlated with both recollection, when controlling for familiarity $[r(33)=.62, p<.001]$, and familiarity, when controlling for recollection $[r(33)=.52, p<.005]$. Moreover, a mean-centered regression $\left[R^{2}=.41, R_{\mathrm{adj}}^{2}=\right.$

Table 3 Experiment 2: Generation rates for three cued recall conditions, the average, and baseline; proportion recall for the three conditions and average recall; zero order correlations between all three
$.37 ; F(2,33)=11.53, p<.001]$ indicated that, consistent with the partial correlations, cued-recall performance was predicted by both recollection $[\beta=.65 ; t(33)=4.49, p<$ $.001]$ and familiarity $[\beta=.50 ; t(33)=3.43, p<.005]$, and multicollinearity measures were within acceptable ranges $(\mathrm{VIF}=1.19, \kappa=6.57)$.

\section{Discussion}

The results from both the correlation and regression analyses demonstrated that recollection was closely related to cued recall. This finding indicates that the ROC-derived estimate of recollection is a sensitive measure that is highly correlated with certain forms of memory. This reassures us that the correlation between conceptual priming and familiarity, but not recollection, in Experiments $1 \mathrm{a}$ and $1 \mathrm{~b}$ could not be explained by the notion that only the familiarity parameter is a sensitive memory measure. Moreover, given that recollection and familiarity were negatively correlated in Experiments 1a and 2, the finding of a significant relationship between recollection and cued recall is inconsistent with the notion that the null relationship in Experiment 1a between recollection and conceptual priming was an artifact produced by collinearity between the recognition parameters. Finally, while the results of Experiment 2 suggest that recollection was strongly related to explicit cued recall, the relationship between recall and familiarity was less clear. The zero-order correlation did not show a significant correlation between familiarity and associative cued recall, but the subsequent partial correlation and regression analyses suggested that familiarity was significantly related to recall. Potential accounts of the latter finding are discussed below.

\section{General discussion}

In the present study, we assessed the relationship between recollection and familiarity estimates, derived from ROC

recall conditions and average recall and $d^{\prime}$, recollection, and familiarity; partial correlations between all three recall conditions and average recall and recollection and familiarity

\begin{tabular}{|c|c|c|c|c|c|c|c|}
\hline & \multirow[t]{2}{*}{$M(S D)$} & \multirow[t]{2}{*}{ Cued Recall $(S D)$} & \multicolumn{3}{|c|}{ Correlation With Recall } & \multicolumn{2}{|c|}{ Partial Correlation } \\
\hline & & & $d^{\prime}$ & $\mathrm{R}$ & $\mathrm{F}$ & $\mathrm{R}$ & $\mathrm{F}$ \\
\hline EncRec & $.49(.12)$ & $.42(.13)$ & $.54^{*}$ & $.42^{*}$ & .19 & $.56^{*}$ & $.44^{*}$ \\
\hline Enc & $.31(.11)$ & $.25(.09)$ & $.59^{*}$ & $.44^{*}$ & .23 & $.59^{*}$ & $.49^{*}$ \\
\hline $\operatorname{Rec}$ & $.36(.12)$ & $.29(.11)$ & $.57^{*}$ & $.38^{*}$ & .22 & $.52^{*}$ & $.44^{*}$ \\
\hline Average & $.39(.11)$ & $.32(.10)$ & $.62^{*}$ & $.45^{*}$ & .24 & $.62^{*}$ & $.51^{*}$ \\
\hline Baseline & $.07(.07)$ & & & & & & \\
\hline
\end{tabular}

EncRec, words presented in both the encoding and recognition phases; Enc, words presented only at encoding; Rec, words presented only in the recognition test. ${ }^{*} p<.05$. 
and remember/know analyses, against measures of conceptual implicit memory. The results from Experiment 1a demonstrated that conceptual priming correlated with ROCbased estimates of familiarity, but not with recollection. The correlations were observed even when we removed participants claiming to have used explicit retrieval strategies, and regardless of whether words had been encoded in an incidental encoding task (i.e., abstract/concrete judgment) and/or during a recognition task. Moreover, additional analysis suggested that the results could not be adequately explained by mediation, suppression, or collinearity effects. Finally, in Experiment $1 \mathrm{~b}$ we replicated this finding using recollection and familiarity estimates derived from the remember/know procedure, demonstrating that the effects generalized across two different measurement methods.

These results support the hypothesis that familiarity and conceptual implicit memory rely on a common underlying process (Henke, 2010; Wagner \& Gabrieli, 1998; Wang et al., 2010; Yonelinas, 2002; but see Voss et al., 2012), and converges with prior studies indicating that conceptual fluency influences familiarity judgments (Rajaram \& Geraci, 2000; Verfaellie \& Cermak, 1999) and that conceptual processing improves subsequent familiarity-based recognition (Ngo, Brown, Sargent \& Dopkins, 2010). The results are also consistent with neuroimaging and patient lesion data suggesting that the perirhinal cortex is involved in both familiarity (Diana et al., 2007; Eichenbaum et al., 2007; Yonelinas et al., 2010) and conceptual priming (O'Kane et al., 2005; Voss et al., 2009; Wang et al., 2010). In light of this convergent evidence, it is likely that the cognitive process that supports familiarity judgments also supports conceptual implicit memory.

It is important to point out that the present results do not imply that there are no differences between familiarity and conceptual implicit memory. Familiarity accounted for $22 \%$ of the variance in conceptual implicit memory in Experiment 1a, while an examination of the zero-order correlations between our three measures of conceptual priming accounted for between $42 \%$ and $53 \%$. This puts an effective ceiling on the amount of variance that the familiarity measure could account for (i.e., approximately half of the variability in priming). This is a substantial portion, but it leaves open the possibility that other processes may also contribute to conceptual implicit memory. For example, the decision processes involved in explicit recognition are quite different from those involved in conceptual implicit tasks, and so it should not be surprising if the tasks engage different processes. The claim that familiarity and conceptual implicit memory are influenced by task-specific processes is supported by imaging studies suggesting important differences in the patterns of activity associated with these two types of tasks (e.g., Donaldson et al., 2001; Voss, Reber, Mesulam, Parrish \& Paller, 2008).
In Experiment 2, recollection significantly predicted associative cued recall. This result demonstrates that the ROCbased estimate of recollection is not inherently less powerful or reliable than the estimate of familiarity. Rather, this is consistent with the idea that recollection and recall rely on a common underlying process (Quamme et al., 2004; Yonelinas, 2002). There also appeared to be a relationship between familiarity and associative cued recall. The zeroorder correlation between familiarity and recall was positive, but it failed to reach statistical significance, and the partial correlation and regression analyses indicated that familiarity and recall were significantly related. Thus, the results are consistent with prior results showing that cuedrecall tasks can be supported at least in part by familiarity (Gruneberg \& Monks, 1974; McCabe et al., 2011).

To what extent are the present conclusions based on the specific manner in which the ROC and remember/know results were analyzed? At the broadest level, the present results are unambiguous in showing a direct relationship between overall recognition memory and conceptual priming. This was shown to be the case in both the ROC and remember/know experiments, in which overall recognition was examined prior to examining the levels of confidence or reports of remembering and knowing. Thus, there is no question that the results are in good agreement with models that assume that a common process contributes to both implicit and explicit memory.

The additional finding that conceptual implicit memory was related to familiarity, but not to recollection, does rely critically on the assumptions used to analyze the confidence and remember/know results. Although a growing literature has validated those estimation procedures (e.g., Yonelinas et al., 2010; but see Wixted, 2007), and the convergence of results across the two methods attests to the robustness of those conclusions, it is useful to examine the results in light of alternative approaches. For example, if one wished to adopt a single-component signal detection model of memory, one would end up with largely the same conclusions. That is, in Experiment 1a, the correlation between $d$ ' measured at the midpoint on the confidence scale was quite large (.44); however, if $d$ ' was measured for the high-confidence responses, then the correlation with priming would decrease to .33. Similarly, in Experiment 1b, the correlation between overall recognition and priming was also quite large (.55), but if $d$ ' was measured for the "remember" responses, the correlation with priming would drop to .39 . Thus, conceptual implicit memory appears to be more strongly correlated with recognition if one includes the weaker items in the analysis (i.e., those items accepted on the basis of familiarity). In contrast, the relationship between recognition and cued recall (i.e., Exp. 2; .62) remained constant when one examined overall recognition with high-confidence recognition (.60). Note that the results can also be examined using a 
signal detection model with two functionally independent memory components (i.e., the unequal-variance signal detection model), and the results of that approach also converge in suggesting that the familiarity strength parameter (i.e., $d^{\prime}$ ) is correlated with conceptual priming (for details of those calculations, see Appendix B).

In summary, we assessed how the processes of recollection and familiarity were related to conceptual implicit memory and associative cued recall. The results demonstrated that conceptual priming is related to familiarity, but not to recollection, whereas cued recall is related to both familiarity and recollection. These results support previous research that has suggested that familiarity and conceptual priming rely on a similar underlying process (Yonelinas, 2002), and they argue against models that postulate that explicit and implicit memory reflect fundamentally separate processes (e.g., Squire, 2004). In addition, the cued-recall results are consistent with research demonstrating that both recollection and familiarity support associative recall (McCabe et al., 2011) and recognition (Starns \& Ratcliff, 2008).

Author note Research for this study was supported by Grants R01 MH59352 and F31 MH096346 from the National Institute of Mental Health, the PBK NCA Graduate Scholarship, and the UC Davis and Humanities Graduate Research Fellowship.. Correspondence concerning this article should be addressed to Wei-chun Wang.

\section{Appendix A}

In general, we have found that recollection and familiarity estimates are not correlated across participants, but that they can become negatively correlated when there are small numbers of trials in the ROC analysis. To determine whether such subsampling could have led to the negative correlation we observed, we randomly generated a set of 48 hypothetical recollection $(M=0.40, S D=0.27)$ and familiarity $(M=$ $1.11, S D=0.62$ ) parameter pairs approximating the mean values observed in Experiment 1a (.37 and .95, respectively), such that across the simulated subjects the recollection and familiarity parameters were not correlated $[r(46)=.00$, $p=.99]$. We then asked what would happen when recognition memory was subsampled? In order to ensure that we used realistic criterion values, we used each participant's Experiment 1a criterion values along with the hypothetical recollection and familiarity parameters to generate the predicted hit and false alarm rates at each criterion for each hypothetical subject. Using these predicted hit and false alarm rates, we created a distribution of 10,000 old and 10,000 new confidence responses for each hypothetical subject that we then randomly subsampled 100 times to obtain 100 sets of 80 old and 80 new trials from each hypothetical subject. Thus, we used hypothetical recollection and familiarity parameters and real criterion values to generate confidence response distributions that approximated the conditions in Experiment 1a.

An examination of the subsampled data showed that we were able to accurately recover the original estimates of recollection $(M=.39, S D=.03)$ and familiarity $(M=$ $1.09, S D=.06)$. However, most importantly, whereas there was no correlation between the unbiased recollection and familiarity parameters used to generate the data, there was a negative correlation between the estimates of recollection and familiarity from the subsampled data $(M=-.25, S D=.13$; $99 \%$ CI $[-.28,-.22])$, similar to the data from Experiments 1a and 2 . These results do not show that subsampling necessarily caused the negative correlation that we observed, but they do verify that such a negative correlation can be expected when examining ROCs under the present conditions. Moreover, further examination of the simulated data indicated that the initial recollection estimates were strongly correlated with the recollection estimates from the subsampled data $(M=.72$, $S D=.09 ; 99 \%$ CI $[.70, .74])$, with the same pattern in familiarity $(M=.80, S D=.07 ; 99 \%$ CI $[.78, .82])$. Thus, it is unlikely that the negative correlation between recollection and familiarity produced a selective correlation between familiarity and conceptual implicit memory.

\section{Appendix B}

To test the generalizability of the present conclusions, we also assessed the ROC-based recognition results using an alternative measurement model: the unequal-variance signal detection (UVSD) model (for a review, see Yonelinas \& Parks, 2007). The model is sometimes referred to as a single-process strength model, but it does require two functionally independent memory parameters in order to account for recognition ROC results of the type seen in the present study (Ratcliff, Sheu, \& Gronlund, 1992; Yonelinas \& Parks, 2007). Fitting this model to recognition ROCs produces estimates of $d^{\prime}$ (strength) and old/new variance ratio (variance). The analysis showed that, in Experiment 1a, conceptual priming did not correlate significantly with either variance $[r(46)=-.21, p=.15]$ or $d^{\prime}[r(46)=.06, p=$ .69]. However, item variance and memory strength were positively correlated to one another $[r(46)=.71 p<.05]$, so, we analyzed the partial correlations, which indicated that variance was negatively correlated with conceptual priming when controlling for $d^{\prime}[r(45)=-.35, p<.05]$, whereas $d^{\prime}$ was positively correlated with priming when controlling for variance $[r(45)=.29, p<.05]$. The mean-centered multiple regression $\left[R^{2}=.13, R_{\mathrm{adj}}{ }^{2}=.09 ; F(2,45)=3.24, p<.05\right]$ also indicated that variance negatively predicted priming $[\beta=-.50, t(45)=-2.51, p<.05]$ whereas $d$ ' positively predicted priming $[\beta=.41, t(45)=2.06, p<.05]$. 
Multicollinearity measures were within acceptable ranges $(\mathrm{VIF}=2.00, \kappa=7.46)$. Thus, these results indicate that familiarity strength was positively related to conceptual implicit memory.

In Experiment 2, cued recall was found to be correlated with both variance $[r(34)=.43, p<.01]$ and strength $[r(34)=$ $.58, p<.001]$. However, because variance and strength are also positively correlated in this experiment $[r(34)=.83, p<$ $.05]$, we again analyzed both the partial correlations and regression, which indicated that variance was not correlated with cued recall when controlling for $d^{\prime}[r(33)=-.12, p=.50]$, whereas $d$ ' was significantly correlated with recall when controlling for variance $[r(33)=.45, p<.05]$. The mean-centered multiple regression $\left[R^{2}=.35, R_{\mathrm{adj}}{ }^{2}=.31 ; F(2,33)=8.76, p<\right.$ $.001]$ also suggested that strength significantly predicted recall $[\beta=.73 ; t(33)=2.86, p<.01]$, whereas variance $\operatorname{did} \operatorname{not}[\beta=$ $-.18 ; t(33)=-0.69$ ]. Again, the multicollinearity measures were within acceptable ranges $(V=3.27, \kappa=10.80)$. The pattern of results produced by the UVSD model is complex, but in general it appears that $d$, a memory strength measure, is positively related to both conceptual implicit memory and associative cued recall. To the extent that strength in this model (i.e., $d^{\prime}$ ) is similar to familiarity, the results of the UVSD-based analysis converge with the finding that familiarity is correlated with conceptual priming and cued recall. However, the behavior of the variance parameter in the model was less clear. The partial correlations suggested that it was negatively related to conceptual priming, but not related to recall. Within the framework of the signal detection model, it is not clear why this parameter would track implicit memory but not explicit memory. However, given that the parameter correlations and collinearity measures are numerically greater in the UVSD analysis relative to the DPSD analysis, these results should be interpreted with caution.

\section{References}

Blaxton, T. A. (1992). Dissociations among memory measures in memory impaired subjects: Evidence for a processing account of memory. Memory \& Cognition, 20, 549-562.

Bowers, J. S., \& Schacter, D. L. (1990). Implicit memory and test awareness. Journal of Experimental Psychology: Learning, Memory, and Cognition, 16, 404-416. doi:10.1037/0278-7393.16.3.404

Challis, B. H., \& Sidhu, R. (1993). Dissociative effect of massed repetition on implicit and explicit measures of memory. Journal of Experimental Psychology: Learning, Memory, and Cognition, 19, 115-127.

Diana, R. A., Yonelinas, A. P., \& Ranganath, C. (2007). Imaging recollection and familiarity in the medial temporal lobe: A threecomponent model. Trends in Cognitive Sciences, 11, 379-386. doi:10.1016/j.tics.2007.08.001

Donaldson, D. I., Petersen, S. E., \& Buckner, R. L. (2001). Dissociating memory retrieval processes using fMRI: Evidence that priming does not support recognition memory. Neuron, 31, $1047-1059$.
Eichenbaum, H., Yonelinas, A. P., \& Ranganath, C. (2007). The medial temporal lobe and recognition memory. Annual Review of Neuroscience, 30, 123-152. doi:10.1146/ annurev.neuro.30.051606.094328

Gabrieli, J. D. E. (1998). Cognitive neuroscience of human memory. Annual Review of Psychology, 49, 87-115.

Gardiner, J. M. (1988). Functional aspects of recollective experience. Memory \& Cognition, 16, 309-313. doi:10.3758/BF03197041

Gruneberg, M. M., \& Monks, J. (1974). "Feeling of knowing" and cued recall. Acta Psychologica, 38, 257-265. doi:10.1016/00016918(74)90010-9

Hamann, S. B. (1990). Level-of-processing effects in conceptually driven implicit tasks. Journal of Experimental Psychology: Learning, Memory, and Cognition, 16, 970-977.

Henke, K. (2010). A model for memory systems based on processing modes rather than consciousness. Nature Reviews Neuroscience, $11,523-532$.

Jacoby, L. L. (1991). A process dissociation framework: Separating automatic from intentional uses of memory. Journal of Memory and Language, 30, 513-541. doi:10.1016/0749-596X(91)90025-F

Levy, D. A., Stark, C. E. L., \& Squire, L. R. (2004). Intact conceptual priming in the absence of declarative memory. Psychological Science, 15, 680-686.

Light, L. L., Prull, M. W., \& Kennison, R. F. (2000). Divided attention, aging, and priming in exemplar generation and category verification. Memory \& Cognition, 28, 856-872.

Mandler, G. (1980). Recognizing: The judgment of previous occurrence. Psychological Review, 87, 252-271. doi:10.1037/0033295X.87.3.252

McCabe, D. P., Roediger, H. L., III, \& Karpicke, J. D. (2011). Automatic processing influences free recall: Converging evidence from the process dissociation procedure and remember-know judgments. Memory \& Cognition, 39, 389-402. doi:10.3758/ s13421-010-0040-5

Mulligan, N. W., \& Stone, M. (1999). Attention and conceptual priming: Limits on the effects of divided attention in the category-exemplar production task. Journal of Memory and Language, 41, 253-28.

Nelson, D. L., McEvoy, C. L., \& Schreiber, T. A. (1998). The University of South Florida word association, rhyme, and word fragment norms. Retrieved from http://w3.usf.edu/FreeAssociation/

Ngo, C. T., Brown, A., Sargent, J., \& Dopkins, S. (2010). Effects of conceptual processing on familiarity-based recognition. Canadian Journal of Experimental Psychology, 64, 67-76.

O'Kane, G., Insler, R. Z., \& Wagner, A. D. (2005). Conceptual and perceptual novelty effects in human medial temporal cortex. Hippocampus, 15, 326-332.

Quamme, J. R., Yonelinas, A. P., Widaman, K. F., Kroll, N. E. A., \& Sauvé, M. J. (2004). Recall and recognition in mild hypoxia: Using covariance structural modeling to test competing theories of explicit memory. Neuropsychologia, 42, 672-691. doi:10.1016/j.neuropsychologia.2003.09.008

Rajaram, S., \& Geraci, L. (2000). Conceptual fluency selectively influences knowing. Journal of Experimental Psychology: Learning, Memory, and Cognition, 26, 1070-1074.

Ratcliff, R., Sheu, C., \& Gronlund, S. D. (1992). Testing global memory models using ROC curves. Psychological Review, 99, 518-535. doi:10.1037/0033-295X.99.3.518

Rotello, C. M., Macmillan, N. A., Reeder, J. A., \& Wong, M. (2005). The remember response: Subject to bias, graded, and not a process-pure indicator of recollection. Psychonomic Bulletin \& Review, 12, 865-873. doi:10.3758/BF03196778

Schacter, D. L., Chiu, C. Y. P., \& Ochsner, K. N. (1993). Implicit memory: A selective review. Annual Review of Neuroscience, 16, $159-182$.

Squire, L. R. (2004). The medial temporal lobe. Annual Review of Neuroscience, 27, 279-306. 
Srinivas, K., \& Roediger, H. L. (1990). Classifying implicit memory tests: Category association and anagram solution. Journal of Memory and Language, 29, 389-412.

Starns, J. J., \& Ratcliff, R. (2008). Two dimensions are not better than one: STREAK and the univariate signal detection model of remember/know performance. Journal of Memory and Language, 59, 169-182. doi:10.1016/j.jml.2008.04.003

Tulving, E. (1985). Memory and consciousness. Canadian Psychologist, 26, 1-12. doi:10.1037/h0080017

Verfaellie, M., \& Cermak, L. S. (1999). Perceptual fluency as a cue for recognition judgments in amnesia. Neuropsychology, 13, 198205. doi:10.1037/0894-4105.13.2.198

Voss, J. L., Hauner, K. K. Y., \& Paller, K. A. (2009). Establishing a relationship between activity reduction in human perirhinal cortex and priming. Hippocampus, 19, 773-778.

Voss, J. L., Lucas, H. D., \& Paller, K. A. (2012). More than a feeling: Pervasive influences of memory processing without awareness of remembering. Cognitive Neuroscience. http://www.tandfonline. com/doi/abs/10.1080/17588928.2012.674935

Voss, J. L., Reber, P. J., Mesulam, M.-M., Parrish, T. B., \& Paller, K. A. (2008). Familiarity and conceptual priming with faces engage distinct cortical networks. Cerebral Cortex, 18, 1712-1719.

Wagner, A. D., \& Gabrieli, J. D. E. (1998). On the relationship between recognition familiarity and perceptual fluency: Evidence for distinct mnemonic processes. Acta Psychologica, 98, 211-223.

Wang, W. C., Lazzara, M. M., Ranganath, C., Knight, R. T., \& Yonelinas, A. P. (2010). The medial temporal lobe supports conceptual implicit memory. Neuron, 68, 835-842.

Wixted, J. T. (2007). Dual-process theory and signal-detection theory of recognition memory. Psychological Review, 114, 152-176. doi:10.1037/0033-295X.114.1.152
Yonelinas, A. P. (1994). Receiver-operating characteristics in recognition memory: Evidence for a dual-process model. Journal of Experimental Psychology: Learning, Memory, and Cognition, 20, 1341-1354. doi:10.1037/0278-7393.20.6.1341

Yonelinas, A. P. (1999). The contribution of recollection and familiarity to recognition and source-memory judgments: A formal dual-process model and an analysis of receiver operating characteristics. Journal of Experimental Psychology: Learning, Memory, and Cognition, 25, 1415-1434. doi:10.1037/02787393.25.6.1415

Yonelinas, A. P. (2001). Consciousness, control, and confidence: The 3 Cs of recognition memory. Journal of Experimental Psychology: General, 130, 361-379. doi:10.1037/00963445.130.3.361

Yonelinas, A. P. (2002). The nature of recollection and familiarity: A review of 30 years of research. Journal of Memory and Language, 46, 441-517. doi:10.1006/jmla.2002.2864

Yonelinas, A. P., Aly, M., Wang, W. C., \& Koen, J. D. (2010). Recollection and familiarity: Examining controversial assumptions and new directions. Hippocampus, 20, 1178-1194.

Yonelinas, A. P., \& Jacoby, L. L. (1995). The relation between remembering and knowing as bases for recognition: Effects of size congruency. Journal of Memory and Language, 34, 622-643. doi:10.1006/jmla.1995.1028

Yonelinas, A. P., Kroll, N. E., Quamme, J. R., Lazzara, M. M., Sauvé, M. J., Widaman, K. F., \& Knight, R. T. (2002). Effects of extensive temporal lobe damage or mild hypoxia on recollection and familiarity. Nature Neuroscience, 5, 1236-1241.

Yonelinas, A. P., \& Parks, C. M. (2007). Receiver operating characteristics (ROCs) in recognition memory: A review. Psychological Bulletin, 133, 800-832. doi:10.1037/0033-2909.133.5.800 